\title{
Monitorización neurofisiológica intraoperatoria del tronco del encéfalo en un caso de cavernoma en protuberancia
}

\author{
R. Rodríguez; J. Molet; S. de Teresa; P. Treserras; P. Clavel; P. Cano; J. Solivera; F. Muñoz y F. Bartumeus
}

Servicio de Neurocirugía. Hospital Universitario de la Santa Creu i Sant Pau. Barcelona.

Resumen

La introducción del control neurofisiológico intraoperatorio ha conseguido minimizar el riesgo funcional quirúrgico en lesiones localizadas en áreas cerebrales funcionales. En la actualidad realizamos control neurofisiológico intraoperatorio para localizar el área motora o sensitiva y el área del lenguaje mediante estimulación cortical, así como de los pares craneales en cirugía del ángulo ponto cerebeloso. La monitorización neurofisiológica durante cirugía del tronco del encéfalo y fosa romboidea está menos instaurada.

La cirugía del tronco del encéfalo implica una cuidadosa selección de los pacientes, dado el alto riesgo de morbilidad y mortalidad asociadas. Por esta razón, los cavernomas de esta región suelen ser tratados de manera conservadora cuando se trata de un hallazgo casual o no son sintomáticos. Sin embargo, la presencia de un sangrado o afectación neurológica inducen a tomar una decisión quirúrgica, dada la mala evolución natural.

Presentamos el caso de una mujer de 29 años, diagnosticada de cavernomas múltiples, que ingresó por cuadro de debilidad motora y déficit sensitivo en hemicuerpo izquierdo. Se realizó TC craneal y RM que mostraba hemorragia protuberancial y se practicó una craniectomía infratentorial y resección de la lesión vascular por línea media, con control neurofisiológico intraoperatorio del VII, VIII, X y XII pares craneales con lectura electromiográfica.

El control neurofisiológico ayudó a decidir el punto de acceso a la lesión que no afloraba a la superficie, minimizar las secuelas postoperatorias y pronosticar precozmente los déficits asociados con el fin de iniciar una rehabilitación precoz.

PALABRAS CLAVE: Cirugía del tronco del encéfalo. Cavernoma. Estimulación cortical intraoperatoria. Mapping neurofisiológico. Fosa romboidea.

Recibido: 01-09-04. Aceptado: 15-11-04 $\overline{\text { Intraoperative neurophysiological monitoring of brain }}$ stem in a case of cavernoma in the pons

Summary

Neurophysiological monitoring during surgery to avoid damaging of eloquent brain areas is a useful tool. We are performing intraoperative neurophysiological test to locate motor, sensitive and speech areas with cortical stimulation and cranial nerves during cerebellopontine cranial base surgery. Neurophysiological monitoring during brain stem surgery has been less described.

Brain stem surgery implies a careful seleccion of patients for surgery given the high risk of morbidity and mortality. For this reason, conservative treatment is usually indicated when an asymptomatic cavernoma is incidentally found. Instead, when bleeding or neurological deficit appear, operative treatment may be indicated and then the goal of surgery is to avoid the disability linked to the natural history.

We present the case of a 29 years old woman with diagnosis of multiple cavernomas. She was admitted at our hospital because she presented weakness and sensitive disturbance of left limbs and dizziness. The CT scan and MRI showed a pontine haemorrhage caused by a cavernous hemangioma. We operated her on using neurophysiological monitoring of VII, VIII, X and XII cranial nerves with electromyographic recordings.

Postoperative disability could be reduced with a better knowledge of entry zone into the brain stem and early physiotherapy.

KEY WORDS: Brain stem surgery. Cavernous hemangioma. Brain mapping. Neurophysiological mapping. Rhomboid fossa.

Introducción

La monitorización neurofisiológica intraoperatoria para la preservación de áreas funcionales cerebrales constituye 


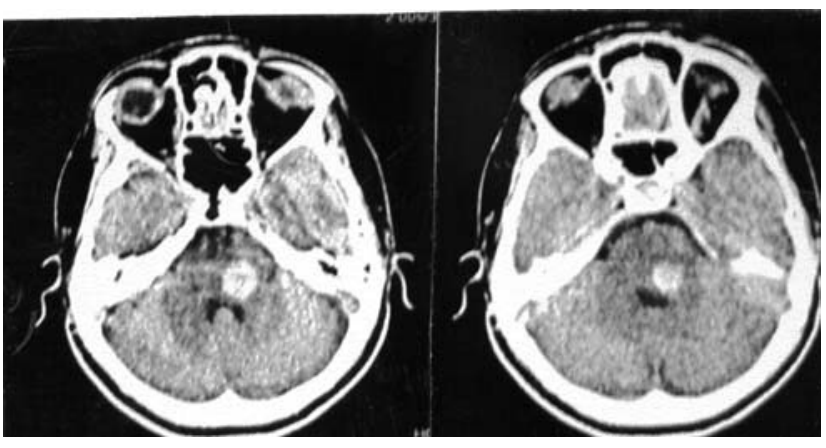

Figura 1. TC craneal axial preopratoria donde puede observarse un hematoma protuberancial en región postero lateral izquierda de la protuberancia, deformando la superficie del IV ventrículo a la altura del colículo facial en la fosa romboidea.

una herramienta de utilidad en gran número de hospitales. La estimulación cortical para definir áreas motora y sensitiva, o localizar regiones en relación con el lenguaje en lóbulo frontal y temporal de hemisferio dominante, para preservarla de la lesión quirúrgica, son de gran utilidad para minimizar las posibles secuelas postoperatorias en cirugía de lesiones próximas a estas localizaciones.

Especial mención merecen, por su dificultad quirúrgica y alta incidencia de morbimortalidad, las lesiones del tronco del encéfalo, donde la gran concentración de núcleos y vías en un pequeño espacio convierte a la cirugía de este territorio en cirugía de alto riesgo.

Con el fin de reducir al máximo las secuelas, sobre todo en lesiones no exofíticas que precisan penetrar en el tronco, la estimulación y monitorización neurofisiológica son de gran utilidad para determinar el lugar de menor riesgo funcional.

En nuestro hospital se han intervenido quirúrgicamente nueve lesiones vasculares del tronco del encéfalo que han debutado con hematoma y sintomatología de tronco ${ }^{44}$. En su mayoría se trataba de lesiones angiográficamente ocultas cuyo estudio histológico revelaba un angioma cavernoso. Sólo el último caso se ha llevado a cabo tras la introducción de la monitorización neurofisiológica intraoperatoria en nuestro centro.

\section{Caso clínico}

Mujer de 29 años de edad, diagnosticada de cavernomas múltiples en 1996, año en que fue intervenida tras hemorragia frontal de angioma cavernoso frontal derecho, con evacuación del hematoma y resección de la lesión vascular. Ese mismo año se objetivó un pequeño angioma protuberancial izquierdo de $3 \mathrm{~mm}$ de diámetro. La paciente permaneció asintomática hasta enero de 2004, cuando ingresa por presentar cefalea y vómitos asociados a hemihipoestesia
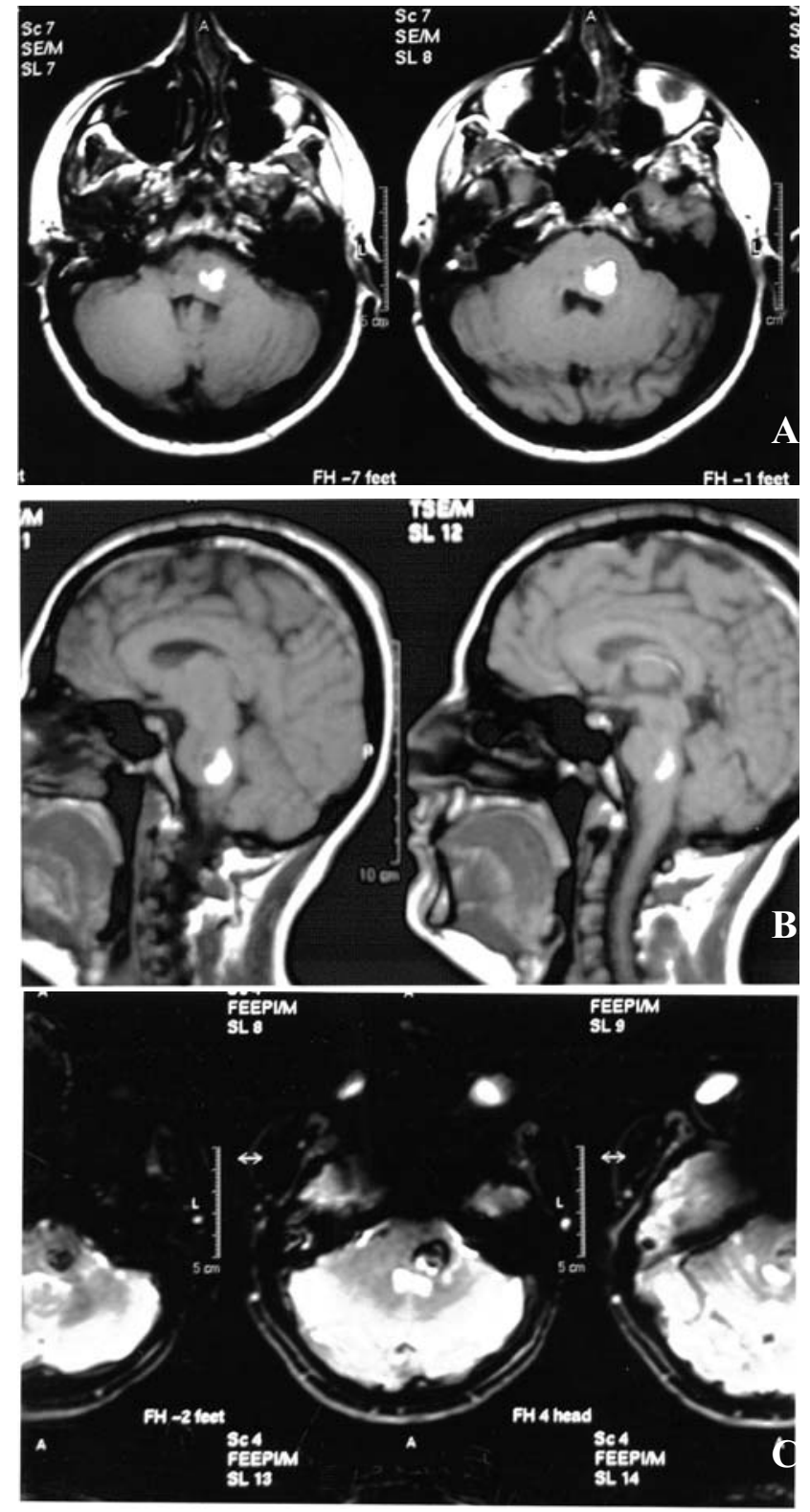

Figura 2. RM preoperatoria observándose un lesión hemorrágica compatible con el sangrado de un angioma cavernoso en protuberancia, deformando la superficie del IV ventrículo a la altura del colículo facial en la fosa romboidea. A) T1 axial, B) T1 sagital, C) Eco de gradiente axial.

con parestesias de hemicuerpo derecho y hemiparesia 4/5 derecha. En la exploración destacaba un nistagmo vertical, una hemiparesia derecha $4 / 5$ y una hemihipoestesia derecha con extinción sensitiva.

La TC craneal realizada al ingreso mostraba un hematoma protuberancial posterior derecho de $20 \mathrm{~mm}$ de diámetro, relacionado con la localización ya conocida del cavernoma (Figura 1).

La RM cerebral confirmaba le presencia de un angioma 

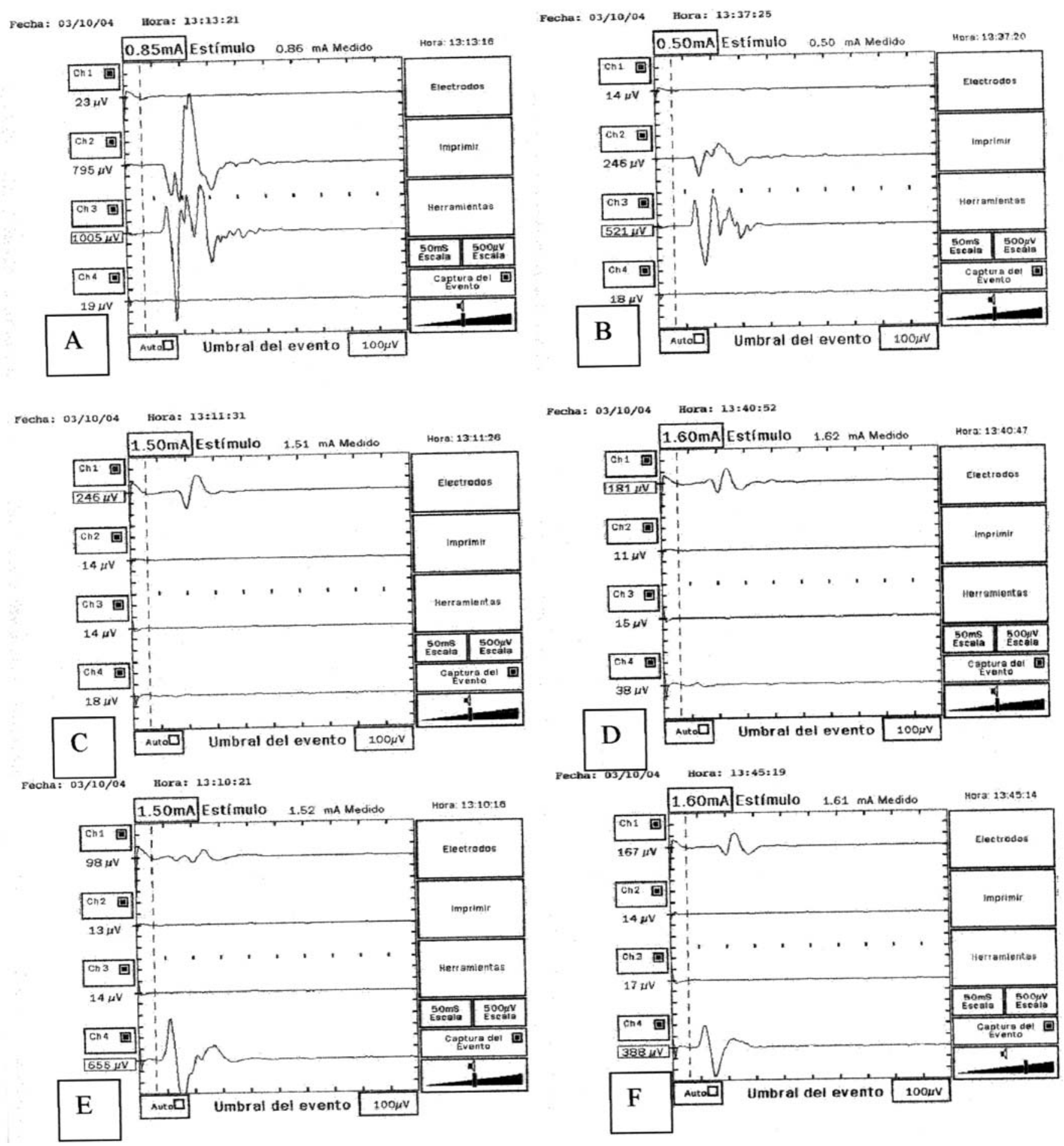

Figura 3. Respuesta electromiográfica tras la estimulación eléctrica de la superficie de la fosa romboidea. A) Facial preoperatorio canal 2: orbicular de los labios; canal 3: orbicular de los ojos B) Facial postoperatorio: obsérvese la reducción de intensidad de la respuesta electromiográfica, sobre todo del canal 2. C) canal 1: X par craneal preoperatorio, D) canal 1: X par craneal postoperatorio. E) canal 4: XII par craneal preoperatorio, F) canal 4: XII par craneal postoperatorio

cavernoso protuberancial con la presencia de hemosiderina perilesional que definían crecimiento y sangrado de la lesión, la cual deformaba el IV ventrículo en la fosa romboidea a la altura del colículo facial izquierdo sin aflorar al 
ventrículo ni a ninguna otra parte de la superficie del tronco del encéfalo (Figuras 2 A y B).

Dada la progresión clínica y radiológica de la lesión se decidió intervenir quirúrgicamente, usando monitorización intraoperatoria que consistió en estimulación de la superficie de la fosa romboidea, para determinar la localización de los núcleos de los pares craneales VII X y XII izquierdos. También se realizaron potenciales auditivos intraoperatorios. Para estimular los núcleos localizados en la fosa romboidea hemos utilizado el sistema de monitorización nerviosa NIM-Response ${ }^{\circledR}$ System (Medtronic, XOMED) aplicando un estímulo monopolar a voltaje constante con pulso monofásico rectangular. La intensidad máxima aplicada fue de $2 \mathrm{~mA}$. La duración del estímulo fue de $100 \mu$ segundos y la frecuencia de $10 \mathrm{~Hz}$. La lectura electromiográfica se realizó con electrodos colocados con una separación de $5 \mathrm{~mm}$ en disposición bipolar para cubrir el área muscular representativa. Los electrodos para monitorizar el nervio facial se colocaron en los músculos orbiculares de la boca y ojo. Para registrar el XII par craneal los electrodos se colocaron en el músculo geniogloso. El registro del X par se realizó con sensores de las cuerdas vocales localizados en el tubo de anestesia.

La paciente fue colocada en decúbito prono realizándose una craniectomía suboccipital y exéresis del arco posterior de $\mathrm{C} 1$. Tras apertura dural y aracnoidea y bajo control microscópico se apreciaba asimetría a la altura del colículo facial izquierdo. Tras identificar anatómicamente los núcleos X y XII, se confirmó su funcionalidad con estimulación intraoperatoria con respuesta electromiográfica positiva (Figura 3). Se localizó el núcleo facial sólo mediante estimulación, encontrándose muy lateral, en el borde entre el ángulo de la fosa romboidea y el pedúnculo cerebeloso medio. El núcleo probablemente se encontraba desplazado lateralmente por el efecto de masa del hematoma que no se visualizaba en superficie. Se realizó incisión pial, se localizó el hematoma que se evacuó, así como el cavernoma causante del mismo. Al finalizar la resección se repitió la monitorización de los núcleos involucrados, obteniendo respuesta electromiográfica en todos, si bien la intensidad del facial, sobre todo de orbicular de los labios, había descendido. Los potenciales auditivos se mantuvieron con la misma respuesta que en el preoperatorio (Figura 3).

En el postoperatorio la paciente presentó paresia transitoria de X y XII, que recuperó íntegramente a las 48 horas y que sospechamos se debió al bloqueo producido por el estímulo eléctrico repetido. También presentó una oftalmoplejía internuclear con síndrome del uno y medio que ha recuperado de manera parcial, persistiendo una paresia nuclear del VI par izquierdo, así como una paresia facial nuclear que ha mejorado progresivamente. En cuanto a vías largas no se observa déficit motor, si bien presenta inestabi- lidad a la marcha, aunque deambula sin ayuda, de manera autónoma. El nivel de conciencia es bueno, con funciones superiores cognitivas conservadas.

\section{Discusión}

El primer caso descrito de evacuación de un hematoma de tronco probablemente secundario a un angioma cavernoso fue publicado por Dandy ${ }^{7,43}$.

El angioma cavernoso es una de las malformaciones vasculares intracraneales más infrecuentes, con una incidencia en necropsias entre 0.39 y $0.53 \% 0^{24,31,33}$. La angiografía en estos pacientes no muestra hallazgos patológicos $^{31}$. Entre el $10-23 \%$ se localizan en la fosa posterior, con predominio de la protuberancia ${ }^{13,19,20,31,37}$. Si estas lesiones sangran pueden ocasionar graves secuelas funcionales. Estudios retrospectivos muestran una mortalidad del 20\% en 30 pacientes tratados de forma conservadora ${ }^{11,43}$.

\section{Indicación quirúrgica}

La cirugía del tronco del encéfalo es muy compleja y comporta un elevado riesgo de secuelas y mortalidad, por lo que la indicación quirúrgica en los angiomas cavernosos que afectan al tronco del encéfalo ha sido controvertida.

Existe consenso general de que los pacientes con angiomas cavernosos incidentales no sean intervenidos quirúrgicamente. Sin embargo, cuando la primera hemorragia ocurre, el tratamiento quirúrgico debería ser considerado, incluso si sólo se presenta con leves déficits neurológicos ${ }^{31}$. Para indicar cirugía parece bastante aceptado que se debe haber producido al menos un episodio de sangrado ${ }^{43}$.

La indicación quirúrgica se basa en el análisis del riesgo de la cirugía en oposición con el tratamiento conserva$\operatorname{dor}^{2,6,10,11,25,27,28,37,38-41,43}$.

El riesgo de sangrado anual en pacientes con cavernomas hallados incidentalmente, asintomáticos es de $2 \%$, mientras que en pacientes sintomáticos es de $6.8-7 \%$ paciente/año $0^{22,31}$.

Algunos autores defienden que tras una hemorragia previa el riesgo de sufrir repetidas hemorragias es mayor ${ }^{1,11,17,26,31,32}$. Porter y cols, describen una incidencia de resangrado de $30 \%$ /persona/año y del $21 \% /$ lesión/año con déficits neurológicos más graves ${ }^{26,31}$. Mathiesen y cols. en una serie de 29 pacientes intervenidos por cavernomas de tronco no consiguieron radicalidad en la exéresis quirúrgica en 4, cuya evolución fue insatisfactoria con la presencia de sucesivos resangrados en todos los casos, con aparición de nuevos déficits permanentes en 3 y muerte en el cuarto. Los 25 pacientes con resección completa siguieron una evolución neurológica más satisfactoria.

De 11 pacientes intervenidos en una serie de 16 
cavernomas de fosa posterior, dos fueron reintervenidos por desarrollo de nuevos síntomas, evidenciándose la existencia de lesiones residuales o de nueva aparición en las proximidades del lecho quirúrgico ${ }^{19}$.

\section{Clínica}

La primera hemorragia se presenta en la mayoría de los pacientes en forma de vértigo, cefalea, y hemihipoestesia; sin embargo, repetidas hemorragias empeoran los déficits neurológicos con trastornos progresivos de la marcha, paresia de pares craneales y de vías largas ${ }^{31}$.

Puesto que en los casos de resangrado las secuelas son más graves e invalidantes y el riesgo de mortalidad es más alto, parece haber acuerdo en la actitud quirúrgica sobre estas lesiones, una vez se ha producido el primer episodio, puesto que la evolución natural de la enfermedad parece peor que el riesgo quirúrgico.

\section{Momento de la cirugía}

El momento óptimo de la cirugía está poco definida, aunque parece que la fase subaguda ${ }^{2,10,31}$ con un retraso de algunos días o semanas tras la hemorragia, cuando el paciente se encuentra en una situación estable sería la más adecuada, siendo el plano de resección más claro, mientras que a los meses el hematoma estará organizado y las estructuras adyacentes se adhieren a una pseudocápsula fibrótica ${ }^{19,21,22}$.

\section{Tratamientos alternativos}

La radiocirugía no ha mostrado efectividad en el tratamiento de los angiomas cavernosos de tronco. Una morbimortalidad y riesgo de resangrado elevadas, así como la presencia de déficits permanentes y complicaciones asociadas a la radiación han limitado esta técnica ${ }^{3,12,15,16,19,22,29,43}$.

El drenaje estereotáctico de los hematomas de tronco puede mejorar temporalmente los déficits neurológicos al reducir el efecto de masa, pero permanecerá el riesgo de resangrado al no resecar el cavernoma ${ }^{4,38,43}$.

\section{Técnica quirúrgica}

La dificultad técnica y la alta concentración de vías y núcleos que pasan por la protuberancia y región bulbomedular, ha derivado en los últimos años en la instauración de la monitorización intraoperatoria neurofisiológica, que junto a un buen conocimiento anatómico, permiten minimizar el riesgo y la severidad de secuelas postquirúrgicas ${ }^{36}$.

En cuanto a la técnica quirúrgica existen diferentes alternativas y vías de acceso dependiendo de la localización del cavernoma: accesos posteriores o posterolaterales, laterales, anterolaterales y anteriores.
En las lesiones supracoliculares, la vía supracerebelosa $^{35,43}$ o supra e infratentorial combinada ${ }^{34,42,43}$ sería aconsejable. Para lesiones de línea media del IV ventrículo sería posible el acceso transvermiano con la consecuente ataxia truncal permanente que podría quedar ${ }^{43}$. Cuando la lesión está más próxima al plano pial lateral protuberancial que a la luz del IV ventriculo es preferible llevar a cabo un abordaje a través de la cisterna del APC ${ }^{19}$. Para las lesiones de la unión pontobulbar, que se presentan en la parte lateral del IV ventriculo, se realizaría un acceso subtonsilar, abriendo la fisura medular, sin retracción del vermis ${ }^{19,38,43}$. Este abordaje ha sido el seleccionado en nuestro caso y estaría indicado para acceder a la plataforma colicular o al cuarto ventrículo. Una vez en la fosa romboidea, la selección del punto de entrada en el tronco puede ser supra o infracolicular, dependiendo de la respuesta neurofisiológica hallada tras estimulación, así como de la localización de la lesión y su relación con núcleos y vías adyacentes ${ }^{36}$.

Las áreas rostral y caudal del colículo facial puede ser incidido con seguridad para entrar en el tronco del encéfalo $^{18,23}$. La dificultad radica en determinar un punto de entrada seguro en el tronco del encéfalo, pues existe riesgo de lesión del núcleo del facial o de su raíz intramedular, así como de los pares craneales bajos condicionada por la pérdida de marcas anatómicas en la superficie del IV ventrículo causada por la distorsión del tronco del encéfalo en lesiones intrínsecas ${ }^{8,9,14,23}$. Tampoco es fácil identificar el trígono formado entre el vago y el hipogloso en pacientes con lesiones de tronco y establecer un punto de entrada seguro entre la estría medular y el obex ${ }^{23}$.

La vía infracolicular se asocia a paresia horizontal de la mirada en el postoperatorio (síndrome del uno y medio) y paresia facial, normalmente transitorias y posible, aunque inusual, afectación de pares craneales bajos que podrían conllevar traqueostomía y gastrostomía ${ }^{36}$. La vía supracolicular presenta en el postoperatorio paresia ocular horizontal (oftalmoplejía internuclear y síndrome del uno y medio), así como paresia del IV par, que también suelen ser transitorias ${ }^{36}$. En nuestro caso, a pesar de la distorsión ocasionada por el efecto de masa, se seleccionó una vía infracolicular, ocasionando en el postoperatorio un síndrome del uno y medio, que se recuperó hasta persistir discreto déficit de la abducción del ojo izquierdo, así como una paresia facial, parcialmente recuperada.

\section{Limitaciones del método}

La presencia de respuesta electromiográfica tras la estimulación de los núcleos motores de los pares craneales, previa a la resección de la lesión del tronco del encéfalo, implica indemnidad de los núcleos y vías implicados. Por el contrario, la presencia de dicha respuesta, tras la resección de la lesión y entrada en el tronco del encéfalo, 
no implica necesariamente su integridad, pudiendo existir lesión del núcleo, de las vías aferentes o eferente, de la raíz intramedular del facial o de las fibras cortico bulbares, siendo la respuesta electromiográfica producto de la difusión de la electricidad a través de las fibras distales, aún cuando el núcleo motor o la raíz intramedular hubiesen sido dañadas intraoperatoriamente ${ }^{23}$. Ello puede limitar en cierto grado el establecer un pronóstico funcional favorable ante respuesta a la estimulación positiva tras la resección. Esto podría explicar los déficits postoperatorios facial y de pares bajos de nuestro caso, aunque estos últimos se recuperaron de manera completa en 48 horas, por lo que el bloqueo eléctrico también podría considerarse como una explicación plausible.

\section{Bibliografía}

1. Alba, T., Tanaka, R., Koike, T., Kameyama, S., Takeda, N., Komata, T.: Natural history of intracranial cavernous malformations. J Neurosurg 1995; 83: 56-59

2. Bertalanffy, H., Gilsbach, J.M., Eggert, H.R., Seeger, W.: Microsurgery of deep-seated cavernous angiomas: report of 26 cases. Acta Neurochir (Wien) 1991, 108: 91-99

3. Bicknell, J.M., Carlow, T.J., Kornfeld, M., Stovring, J., Turner, P.: Familial cavernous angiomas Arch Neurol 1978; 35: 871-876.

4. Bosch, D.A., Beute, G.N.: Successful stereotaxic evacuation of an acute pontomedullary hematoma: Case report. J Neurosurg 1985, 2: 153-156

5. Christian Strauss, C., Romstöck, J., Nimsky,C., Fahlbusch, R.: Intraoperative identification of motor areas of the rhomboid fossa using direct stimulation. J. Neurosurg, 1993, 79: 393-399

6. Chyatte, D.: Vascular malformations of the brain stem. J Neurosurg 1989; 70: 847-852.

7. Dandy, W.E.: The Brain. Harper and Row, New York, 1969.

8. Epstein, F., McCleary, E.L.: Intrinsic brain-stem tumors of childhood: Surgical indications. Neurosurgery 1986; 64: 11-15.

9. Epstein, F.J., Farmer, J.P.: Brain-stem glioma growth patterns. J Neurosurg 1993; 78: 408-412.

10. Fahlbusch, R., Strauss, C., Huk, W., Rockelein, G., Kompf, D., Ruprecht, K.W.: Surgical removal of pontomesencephalic cavernous hemangiomas. Neurosurgery 1990, 26: 449-457.

11. Fritschi, J.A., Reulen, H.J., Spetzler, R.F., Zabramski, J.M.: Cavernous malformations of the brain stem. A review of 139 cases. Acta Neurochir (Wien) 1994, 130: 35-46.

12. Karlsson, B., Kilhstrom, L., Lindquist, C., Encson, K., Steiner, L.: Radiosurgery for cavernous malformations. J Neurosurg 1998; 88: 293-297.

13. Kashiwagi, S., van Loveren, H.R., Tew, J.M., Wiot,
J.G., Weil, S.M., Lukin, R.A.: Diagnosis and treatment of vascular brain-stem malformations. J Neurosurg 1990; 72: 27 34.

14. Katsuta, T., Morioka, T., Fujii, K., Fukui, M.: Physiological localization of the facial colliculus during direct surgery on an intrinsic brain stem lesion. Neurosurgery 1993; 321: 861-863.

15. Kondziolka, D., Lunsford, L.D., Coffey, R.J., Bissonette, D.J., Flickinger, J.C.: Stereotactic radiosurgery of angiographically occult vascular malformations: Indications and preliminary experience. Neurosurgery 1990; 27: 892-900.

16. Kondziolka, D., Lunsford, L.D., Flickinger, J.C., Kestle, J.R.W.: Reduction of hemorrhage risk after stereotactic radiosurgery for cavernous malformations. J Neurosurg 1995; 83: 825-831.

17. Kondziolka, D., Lunsford, L.D., Kestle, J.R.: The natural history of cerebral cavernous malformations. J Neurosurg 1995, 83: 820-824.

18. Kyoshima, K., Kobayashi, S., Gibo, H., Kuroyanagi, T.: A study of safe entry zones vía the floor of the fourth ventricle for brain-stem lesions: Report of three cases. J Neurosurg 1993, 78: 987-993.

19. Lobato, R.D., Gómez, P.A., Alday, R., Rivas, J.J., Ortega, J.M., Domínguez, J., Sandoval, H., Cabrera, A., Madero, S., Eyerbe, J.: Cavernomas del tronco cerebral y el cerebelo. Estudio de 16 casos sintomáticos. Neurocirugía 1994; 5: 221-229.

20. Lobato, R.D., Pérez, C., Rivas, J.J., Cordobés, F.: Clinical, radiological and pathological spectrum of angiographically occult intracranial vascular malformations. J Neurosurg 1988; 68: 518-531.

21. Mangiardi, J.R.: The surgical management of brainstem hematomas. Perspectives Neurolog Surg 1991; 2: 33-45.

22. Mathiesen, T., Edner, G., Kihlström, L.: Deep and brainstem cavernomas: a consecutive 8-year series. J. Neurosurg, 2003; 99: 31-37.

23. Morota, N., Deletis, V., Epstein, F.J., Kofler, M., Abbott, R., Lee, M., Ruskin, K.: Brain stem mapping: Neurophysiological localization of motor nuclei on the floor of the fourth ventricle. Neurosurgery, 1995; 5: 922-930.

24. Otten, P., Pizzolato, G.P., Rilliet, B., Bemey, J.: A propos de 131 cas d'angiomes caverneux (cavernomas) du systéme nerveux central reérés par I'analyse rétrospective de 24535 autopsies. Neurochirurgie 1989; 35: 82-83.

25. Ondro, S.L., Doty, J.R., Mahla, M.E., George, E.D.: Surgical excision of a cavernous hemangioma of the rostral brain stem: Case report. Neurosurgery 1988, 23: 490-493.

26. Porter, R.W., Detwiler, P.W., Spetzler, R.F., Lawton, M.T., Baskin, J.J., Derksen, P.T., Zabramski, J.M.: Cavernous malformations of the brainstem: experience with 100 patients. J Neurosurg 1999; 90: 50-58.

27. Pozzati, E., Giuliani, G., Nuzzo, G., Poppi, M.: The growth of cerebral cavernous angiomas. Neurosurgery 1989; 
25: 92-97.

28. Pozzati, E., Gaist, G., Poppi, M., Morrone, B., Padovani, R.: Microsurgical removal of paraventricular cavernous angiomas. J Neurosurg 1981; 55: 308-311.

29. Robinson, J.R., Awad, I.A., Little, J.R.: Natural history of the cavernous angioma. J Neurossurg 1991; 75: 709-714.

30. Rigamonti, D., Drayer, B.P., Johnson, P.C., Hadley, M.N., Zabramski, J., Spetzler, R.F.: The MRI appearance of cavernous malformations (angiomas). J Neurosurg 1987, 67: 518-524.

31. Sandalcioglu, I.E. Wiedemayer, H., Secer, S., Asgari, S., Stolke, D.: Surgical removal of brain stem cavernous malformations: Surgical indications, technical considerations, and results. J Neurol, Neurosurg \& Psychiatry, 2002; 72: 351-355.

32. Sakai, N., Yamada, H., Tanigawara, T., Asano, Y., Andoh, T., Tanabe, Y., Takada, M.: Surgical treatment of cavernous angioma involving the brainstem and review of the literature. Acta Nerochir (Wien). 1991; 113: 138-143.

33. Sarwar, M., Mc Cormick, W.F.: Intracerebral venous angioma. Case report and review. Arch Neurol 1979; 35: 323326.

34. Sekhar, L.N., Goel, A.: Combined supratentorial and infratentorial approach to large pineal-region meningioma. Surg Neurol 1992; 37: 197-201.

35. Stein, B.M.: The infratentorial supracerebellar approach to pineal lesions. J Neurosurg 1971; 35: 197-202.

36. Strauss, C., Romstöck, J., Fahlbusch, R.: Pericollicular approaches to the rhomboid fossa. Part II. Neurophysiological basis. J Neurosurg 1999; 91: 768-775.

37. Voigt, K., Yasargil, M.G.: Cerebral cavernous haemangiomas or cavernomas. Incidence, pathology, localization, diagnosis, clinical features and treatment: review of the lite- rature and report of an unusual case. Neurochirurgia 1976; 19: 59-68.

38. Weil, S.M., Tew, J.M.: Surgical management of brain stem vascular malformations. Acta Neurochir (Wien) 1990, 105: 14-23.

39. Yasargil, M.G,: Microneurosurgery IIIB. AVM of the Brain. Thieme, Stuttgart, 1988: 405-438.

40. Yoshimoto, T., Suzuki, J.: Radical surgery on cavernous angioma of the brainstem. Surg Neurol 1986; 26: 72-78.

41. Zimmerman, R.S., Spetzler, R.F., Lee, K.S., Zabramski, J.M., Hargraves, R.W.: Cavernous malformations of the brain stem. J Neurosurg 1991; 75: 32-39.

42. Ziyal, I.M., Sekhar, L.N., Salas, E., Olan, W.J.: Combined supra and infratentorial transsinus approach to large pineal region tumors. J Neurosurg 1998; 88: 1050-1057.

43. Ziyal, I.M., Sekhar, L.N., Salas, E., Sen, C.: Surgical management of cavernous malformations of the brain stem. $\mathrm{Br}$ J Neurosurg 1999; 13: 366-375.

44. Zurita, B., Ramírez, R., Oliver, B., Molet, J., Tresserras, P., Parés, P., Bartumeus, F.: Malformaciones vasculares intrínsecas del tronco cerebral. Tratamiento quirúrgico. Neurocirugía 1992; 3: 105-109.

Rodríguez, R.; Molet, J.; de Teresa, S.; Treserras, P.; Clavel, P.; Cano, P.; Solivera, J.; Muñoz, F.; Bartumeus, F.: Monitorización neurofisiológica intraoperatoria del tronco del encéfalo en un caso de cavernoma en protuberancia. Neurocirugía 2005; 16: 117-123.

Correspondencia postal: Rodrigo Rodríguez. Servicio de Neurocirugía. Hospital Universitario de la Santa Creu i Sant Pau. Sant Antoni María i Claret, 167. 08025 Barcelona. 